\title{
Correspondence
}

Correspondents should note that space is limited and shorter letters have a greater chance of publication. The Editors reserve the right to cut letters and also to eliminate multitudinous references. Please try to be concise, strictly relevant and interesting to the reader, and check the accuracy of all references in Journal style.

\section{RENAL ENZYMES IN LITHIUM TREATMENT} DEAR SIR,

We read with interest the recent paper by Garvey et al (Journal, October 1982, 141, 420-2) on the use of renal enzymes to evaluate nephrotoxicity in lithiumtreated patients. We would like to draw the attention of the authors to an earlier paper of ours (Acta Psychiatrica Scandinavica (1980), 62, 343-355) in which we also measured NAG* excretion in a larger group of patients (101) who had been on sustained release lithium carbonate for between 1 and 12.5 years. This study included also a group of patients with affective disorders who had never received lithium, as well as a similar control group to that used by Garvey $e t$ al. NAG was used as a measure of renal damage, but we also carried out a range of tests of renal function. We were able to conclude from the more extensive study we carried out that there was little evidence of serious renal functional impairment attributable to lithium.

Since in Garvey et al's study the mean NAG values for the chronic lithium group fell in the normal range, and 20 per cent of the chronic lithium patients who had evidence of renal damage are included in the mean NAG value, the inclusion of the actual NAG value for these patients would have been more satisfactory. Also, the age of the patients with abnormal NAG excretion would be of interest, since the normal level of NAG excretion in individuals over sixty years of age should be corrected for lower creatinine excretion which accompanies the fall in muscle mass with age.

* $\mathrm{N}$-acetyl-glucosaminadase

\section{R. G. Price}

Queen Elizabeth College,

A. COPPEN

(University of London),

Camden Hill Road,

London W8 7AH

\section{Dr Garvey and associates reply}

Eleven affectively disordered patients experienced a NAG level greater than 100 units/mg creatinine. Their ages and NAG levels are presented in the accompanying Table. Three of the eleven patients were sixty or older. The mean age of the eleven patients with an elevated NAG was 44 . The mean age for the entire sample of 65 affectively disordered patients was 41 . Thus age does not appear to be a significant factor in the observed NAG elevations.

TABLE

Affectively disordered patients experiencing NAG elevations

\begin{tabular}{cc}
\hline \multicolumn{2}{c}{ Patients starting lithium treatment } \\
\hline Age & NAG level \\
\hline 23 & 110 \\
64 & 118 \\
29 & 119 \\
27 & 116 \\
\hline \multicolumn{2}{c}{ Chronic lithium patients } \\
\hline Age & NAG level \\
\hline 48 & 135 \\
52 & 103 \\
51 & 103 \\
60 & 113 \\
67 & 112 \\
28 & 205 \\
\end{tabular}

The elevated NAG levels were all between 100 and 205 units. It should be emphasized that none of our 21 controls ( 2 samples per subject) experienced a NAG level greater than 100 units. There were only four NAG levels from 42 control samples that were above 70 units. The highest of these was 85 units. Thus, while the NAG elevations reported in eleven affectively disordered patients were only mildly elevated, we did not observe a single example of this in 42 control samples.

As in the Coppen et al paper (1980) we too found no significant difference in the mean NAG levels between lithium-treated affectively disordered patients and controls. Mean values may however, be misleading. In our paper we offer data from other reports that suggest there may exist a subgroup of approximately 15 per cent of affectively disordered patients with a mild associated renal disorder. If this is true, then the 85 per cent of affectively disordered patients with no associ- 